\title{
Cadaveric Bilateral Transhumeral Upper Limb Transplantation: Journey to the Next level
}

\author{
Kumar S Dinesh ${ }^{1} \quad$ Abhinav Aggarwal ${ }^{1}$ Ravi Kumar Chittoria ${ }^{1} \quad$ Friji Meethale Thiruvoth ${ }^{1}$ \\ Devi Prasad Mohapatra ${ }^{1}$ Vinayak Chavan ${ }^{1}$ Saurabh Gupta ${ }^{1}$ Chirra Likhitha Reddy
}

1Department of Plastic Surgery, Jawaharlal Institute of Postgraduate Medical Education and Research (JIPMER), Pondicherry, India
Address for correspondence Ravi Kumar Chittoria, MBBS, MS, MCh, Department of Plastic Surgery, Jawaharlal Institute of Post Graduate Medical Education and Research (JIPMER), Puducherry 605006, India (e-mail: drchittoria@yahoo.com).

\begin{abstract}
Keywords

- transhumeral hand transplant

- vascularized composite allotransplant

- above elbow transplant

Organ transplant has, arguably, been the pinnacle of the advancement in medical science so far and vascularized composite allotransplantation has been the most recent addition to it. The scope of vascularized tissue allotransplantation has been widening with each passing day and more and more reports regarding the safety and efficacy of the procedure have been described.

Due to the limited available literature on the management of the complications and implications of the hand transplant, the importance of each report on the procedure is paramount.

A mid-arm allotransplant is a challenge in terms of expected motor recovery, and many controversies exist over the efficacy of the procedure altogether. Moreover, our case was complicated by a long ischemia time due to logistic reasons.

We share our experience of a transhumeral upper limb allotransplantation, the complications associated with it along with the early postoperative results at 10 months follow up.
\end{abstract}

\section{Introduction}

Composite tissue transplantation or reconstructive transplantation is a controversial topic. With more and more centers all over the world performing the procedure, it has become more acceptable.

The choice between functional disability and a lifetime of immunosuppression and its complications is a difficult one; however, it is the best possible attempt to restore one's quality of life.

The procedure is a challenging one and due to the relative lack of experience, it is not a common practice. It requires a multidisciplinary team effort involving hand surgeons, plastic surgeons, physiotherapists, psychiatrists, anesthetists, immunologists, psychologists, and occupational therapists.

Due to hand transplantation being a relatively recent procedure, there is a lack of set protocols and literature on the physiology and management of the complications. ${ }^{1}$
This is our second experience with the procedure following one successful reconstructive upper limb transplant at proximal forearm level. ${ }^{2}$

Above elbow amputations have classically not been considered an ideal candidate for transplantation due to the concerns of graft versus host disease (GVHD) due to the amount of bone marrow transplanted, the quality of nerve regeneration and inadequate regeneration of hand function. ${ }^{3}$

In this case, considering the profound impairment in the quality of life of an above-elbow amputee and success results in reimplantations at this level, the procedure was considered. The recipient was well motivated and understood the risks and complications involved. The procedure was done in November 2018 and early postoperative results are reported herein.

\section{Case Report}

A 31-year-old gentleman presented with a history of right proximal forearm amputation and left transhumeral published online April 1, 2020
DOI https://doi.org/ 10.1055/s-0040-1708115 ISSN 0970-0358.
(C)2020 Association of Plastic Surgeons of India
License terms

$($ () (1) $\Theta \circledast$ 
amputation following crush injury of both upper limbs in November, 2015. The patient presented to our hospital for bilateral upper limb transplantation ( - Fig. 1).

The recipient was not a known case of diabetes mellitus, hypertension, or any other known comorbidities.

On examination, on the right side, the amputation level was below elbow with the distance between the acromion process to the lowermost part of the stump to be $37 \mathrm{~cm}$.

On the left side, the amputation level was above elbow at mid-arm with the distance between the acromion process to the lowermost part of the stump to be $17 \mathrm{~cm}$ ( - Fig. 2).

The patient underwent surgery (B/L upper limb transplantation) in November 19, 2018, after 6 months on the waiting list.

The donor was a fully HLA matched traumatic brain dead 23-year-old man of similar skin phenotype and color.

The donor limb harvest was done in a private hospital in Bangalore, around $300 \mathrm{~km}$ away from our hospital. A surgical team was constituted the night before and immediately travelled to Bangalore. The harvest was completed at proximal arm level on both sides and preserved by infusing histidine-tryptophan-ketoglutarate solution and stored in an icebox.

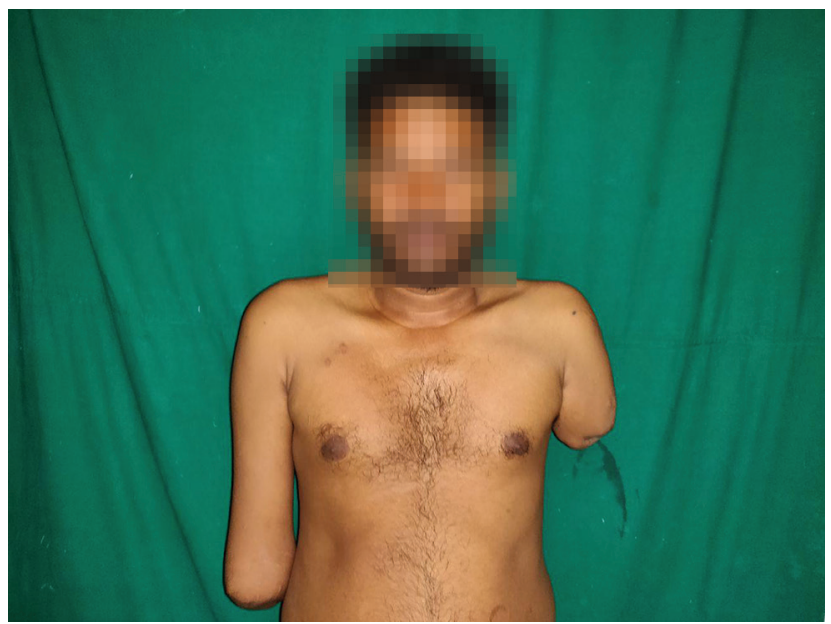

Fig. 1 Recipient preoperative.
The limb was transported from Bangalore to Puducherry by road. The facility of an air-ambulance was not available and a green corridor was constituted.

Due to a bony stump of only $2.5 \mathrm{~cm}$ below the right elbow, the decision was made to revise the amputation at the distal arm level and transhumeral transplantation was done for both upper limbs.

Induction therapy was given with antithymocyte globulin before surgery and immunotherapy comprising of prednisolone, mycophenolate mofetil, and tacrolimus.

\section{Transplantation Surgery}

Transplantation was done in November, 2018, and the procedure lasted for 12 hours.

Simultaneous recipient site preparation was started and all structures tagged. Median, ulnar, and radial nerve neuromata were resected bilaterally. Bone was plated, and dorsal structures were repaired. The total ischemia time was 9 hours (6 hours cold ischemia time and 3 hours warm ischemia time). Due to a long ischemia time, the standard technique of artery last was not followed and vascular anastomosis was done early and it resulted in a large volume blood loss for which transfusions were given. Due to intraoperative hemodynamic fluctuations 9 units of leuco-reduced packed red blood cells (PRBCs), 9 units fresh frozen plasma (FFP), and 5 units of platelets were transfused. The sequence of the structures repaired was bone, triceps tendon, brachialis, cephalic vein, deep veins brachial artery, biceps tendon, and the nerves.

\section{Postoperative Period}

Postoperative immunosuppression was started with mycophenolate mofetil and tacrolimus. The patient had myoglobinuria and limb edema in the initial postoperative period.

A summary of the postoperative period is as follows:

\section{First Week}

The patient had myoglobinuria and massive blood transfusion-related complications such as hemolysis, and hypocalcemia in the early postoperative period. Covering of fasciotomy wound with a split skin graft was done.

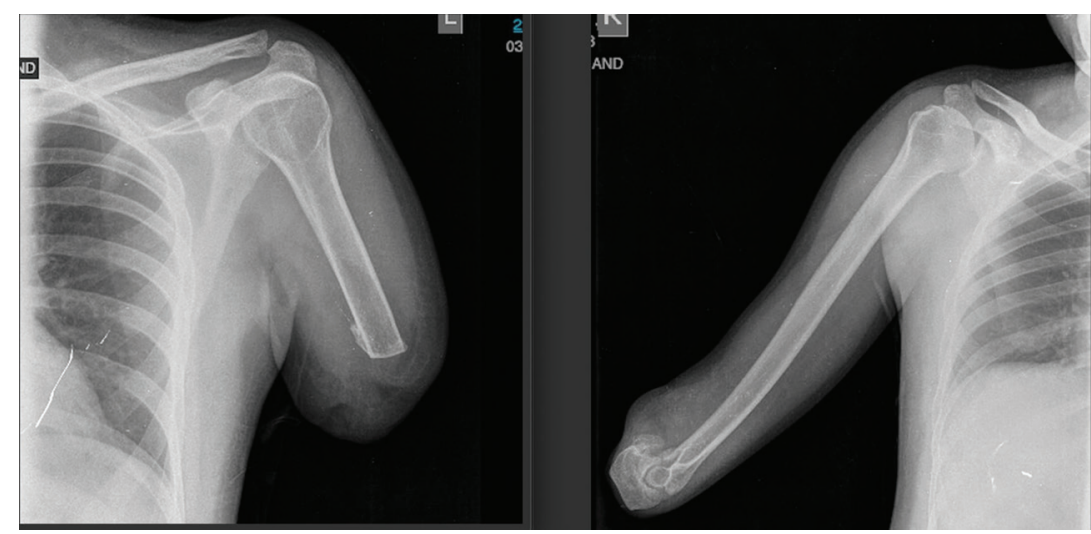

Fig. 2 Left and right upper limb X-ray views of the recipient (preoperative). 
Diabetes (new-onset diabetes after transplant [NODAT]) was noted in the first week and endocrinology opinion was taken, and accordingly insulin was started which was continued throughout his hospital stay.

There was persistent edema in the limb with wound soakage. Negative pressure wound therapy (NPWT) was applied.

\section{Second Week}

Dehiscence of the wound over medial arm left side happened in the second week. Vacuum assisted closure (VAC) dressing was started and the wound recovered well. The first episode of acute rejection was confirmed by biopsy and treated with prednisolone therapy and titration of the tacrolimus. There was persistent proximal arm edema, which resolved by week 3 .

\section{Fifth Week}

Gradual tapering of tacrolimus dose was started.

\section{Sixth to Tenth Week}

Physiotherapy was started. Further tapering of tacrolimus dose was done.

\section{Eleventh Week to Thirteenth Week}

The patient developed a second episode of transplant rejection reaction ( - Fig. $\mathbf{3}$ ), swelling of the limb, the appearance of skin lesions and fever, for which plasmapheresis and FFP were given.

Pulse intravenous immunoglobulin (IVIG) therapy was started and the patient responded well.

\section{Fifteenth to Seventeenth Week}

This period remained uneventful. The patient was started on vigorous physiotherapy including muscle electrostimulation and passive stretching with active goal-oriented exercises.

\section{Eighteenth Weeks to Twenty-Eight Weeks}

Physiotherapy was intensified and results were documented. Wrist flexion and finger flexion and extension (power $3^{+}$) were noted in the right limb with sensations present till the base of fingers with Tinel's sign positive.

\section{Results}

The patient had two biopsy-proven episodes of acute antibody-mediated rejection for which plasmapheresis and IVIG therapy was given to which the patient responded well. He was followed up weekly for the first 3 months and twice a month thereafter, to assess for rejection reaction, opportunistic infection, drug compliance, and record improvement in motor and sensory system of both transplanted upper limbs.

Regular physiotherapy was continued as scheduled and all results were noted.

The patient has grade $4+$ power at the level of the elbow on the right with full sensations up to the base of fingers with advancing Tinel's sign ( - Fig. 4). A video at 7 months postoperative shows movement at the elbow in the right limb (-Video 1). A video at 10 months postoperative shows the ability to lift paper with the thumb and index finger ( $\mathbf{- V i d e o} 2$ ).

There are movements (grade $3+$ ) of the long flexors of the wrist and fingers. The movements at the wrist and elbow are demonstrated in the videos at 7 months post-operative.

( - Videos 3 and 4 )

On the left upper limb, there is a gain of full sensations at the level of mid-forearm and flickering movements of the flexors of the wrist.

The patient is continuing the rehabilitation program as planned before.

\section{Discussion}

The first hand transplant was done in France in $1998 .{ }^{4}$ Since, then multiple reports of hand transplantation have been reported with good to moderate functional results. ${ }^{15,6}$

The scope of composite allotransplant has been expanded to include face transplant as well but our experience with the same is limited. ${ }^{4}$

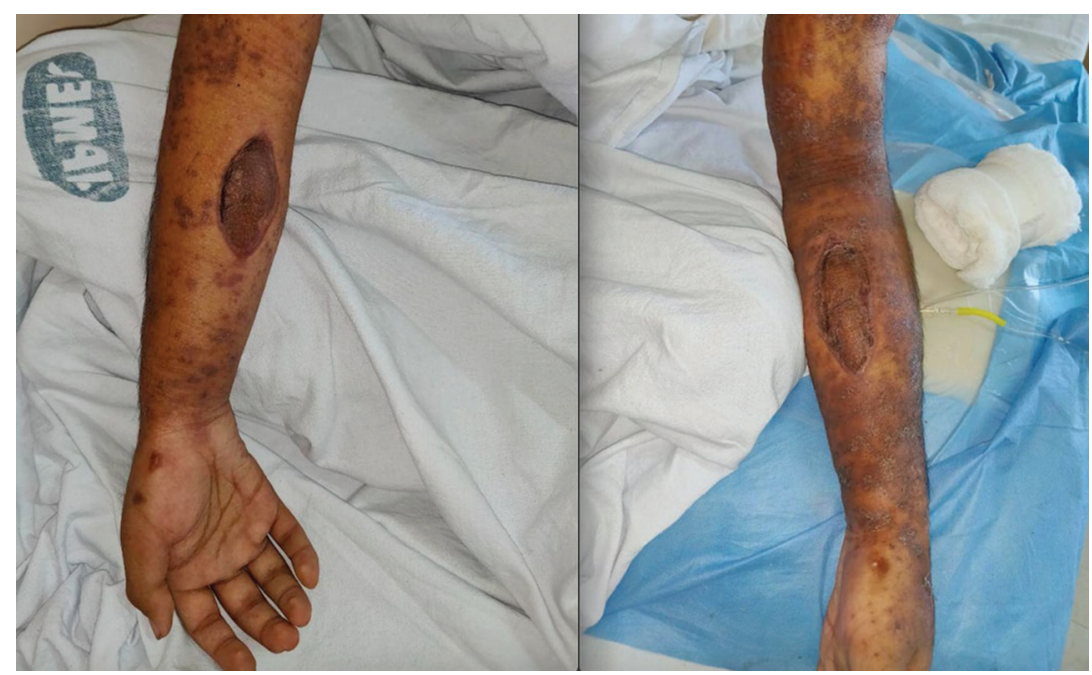

Fig. 3 Second episode of rejection, in right and left upper limb, respectively. 


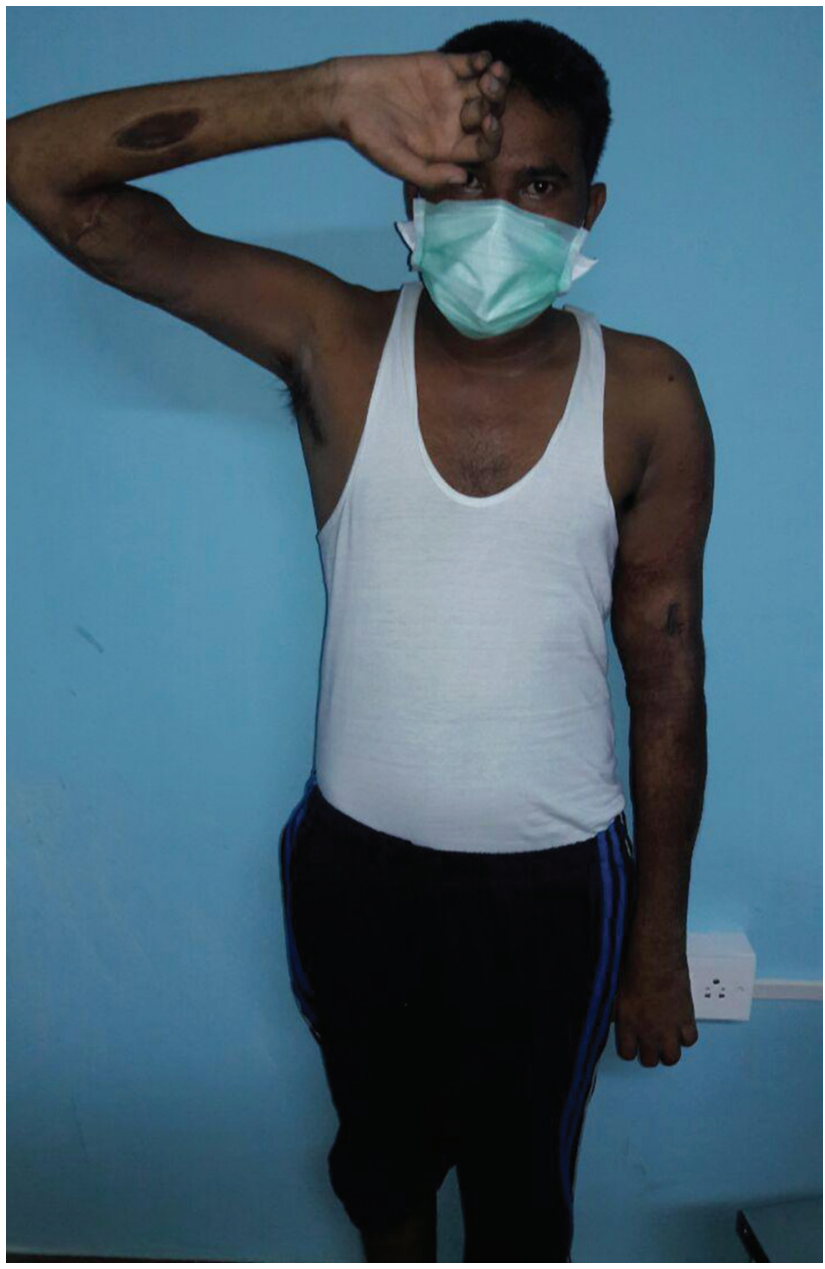

Fig. 4 At 6 months postoperative.

\section{Video 1}

Elbow flexion and extension in right limb being demonstrated at 7 months postoperative. Online content including video sequences viewable at: www.thieme-connect.com/products/ ejournals/html/10.1055/s-0040-1708115.

\section{Video 2}

A Video at 10 months postoperative shows the ability to lift paper with the thumb and index finger. Online content including video sequences viewable at: www.thieme-connect.com/ products/ejournals/html/10.1055/s-0040-1708115.

\section{Video 3}

Wrist flexion and extension in right limb being demonstrated at 7 months postoperative. Online content including video sequences viewable at: www.thieme-connect.com/products/ ejournals/html/10.1055/s-0040-1708115.

\section{Video 4}

Finger flexion and extension in right limb being demonstrated at 7 months postoperative. Online content including video sequences viewable at: www.thieme-connect.com/products/ ejournals/html/10.1055/s-0040-1708115.

The risk versus benefit analysis in hand transplant is doubtful; however, there is a universal agreement for its usefulness in bilateral amputees. The authors experience with one previous procedure was highly successful with gratifying results. $^{2}$

Logically, the higher the level of transplantation, the poorer is the functional result due to limited motor reinnervation. This is especially true in transhumeral transplantations as, there are higher chances of rejection due to high antigenic load.

The relative rapid regain of sensory nerve function may be due to the enhancement of nerve generation by immunosuppressive drugs, that is, calcineurin inhibitors in particular. ${ }^{7}$ A similar finding has been reported by Cavadas et al in their case report as well. ${ }^{3}$

The immunosuppression treatment protocol has been different in different case reports with equal results. Most case reports on hand transplantation have reported multiple episodes of rejection in the immediate perioperative period.

Following IVIG and plasmapheresis for the second episode of rejection, the improvement was dramatic with a resolution of skin lesions within 48 hours.

The prednisolone used to treat the first rejection resulted in severely deranged glycemic control and was not well tolerated. A need for the development of a steroid-sparing protocol is needed.

Long ischemia time ( 9 hours) due to the transportation of the donor limbs lead to a stormy early postoperative course with myoglobinuria and massive blood transfusion-related complications; yet, in the long term, has a good functional result.

It also brings into attention the aspect that lack of air ambulance facility, especially in organ transportation, leads to life-threatening complications and this is something that the administration of the developing countries must take seriously.

Considering that already at 7 months post transplant, there is a motor recovery in the long flexors and regeneration of sensory nerves till the base of the fingers in one limb, the progress is astounding and subsequently, the longer follow-up results will be reported.

It must be noted that these are early postoperative results following the transplant and the final functional gain will be noted and published subsequently but the early postoperative results are indicative of the efficacy of the procedure.

\section{Financial Support and Sponsorship}

None. 


\section{Ethical Approval and Consent to Participate}

Due consent was obtained from the patient involved to use the details and picture of the patient for publication purpose according to Helsinki Declaration.

Ethical approval was taken from the department ethics committee for publishing the above content.

Prior to the procedure, National Organ and Tissue Transplantation Organization (NOTTO) certification and approval was obtained prior to undertaking the procedure and ethical approval was taken prior to procedure as well.

\section{Conflicts of Interest}

All authors declared that there are no conflicts of interest.

\section{Acknowledgments}

None.

\section{References}

1 Shores JT, Brandacher G, Lee WP. Hand and upper extremity transplantation: an update of outcomes in the worldwide experience. Plast Reconstr Surg 2015;135(2):351e-360e

2 Sivakumar DK, Chavan V, Chittoria R, et al. Cadaveric bilateral proximal forearm allotransplantation. Ann Plast Surg 2019;82(5):574-576
3 Cavadas PC, Ibáñez J, Thione A, Alfaro L. Bilateral transhumeral arm transplantation: result at 2 years. Am J Transplant 2011;11(5):1085-1090

4 Petruzzo P, Lanzetta M, Dubernard JM, et al. The international registry on hand and composite tissue transplantation. Transplantation 2010;90(12):1590-1594

5 Shores JT, Malek V, Lee WPA, Brandacher G. Outcomes after hand and upper extremity transplantation. J Mater Sci Mater Med 2017;28(5):72

6 Shores JT, Higgins JP, Lee WP. Above-elbow (supracondylar) arm transplantation: clinical considerations and surgical technique. Tech Hand Up Extrem Surg 2013;17(4):221-227

7 Cottrell BL, Perez-Abadia G, Onifer SM, et al. Neuroregeneration in composite tissue allografts: effect of low-dose FK506 and mycophenolate mofetil immunotherapy. Plast Reconstr Surg 2006;118(3):615-623, discussion 624-625 